\title{
Resistant hypertension and cardiovascular disease mortality in the US: results from the National Health and Nutrition Examination Survey (NHANES)
}

\author{
Katerina R. Kaczmarski ${ }^{1^{*}}$ (D) Stephen M. Sozio ${ }^{1,2}$, Jingsha Chen ${ }^{2,3}$, Yingying Sang ${ }^{2,3}$ and Tariq Shafi ${ }^{1,2,4}$
}

\begin{abstract}
Background: Apparent treatment-resistant hypertension (aTRH) is a common condition associated with risk of cardiovascular events. However, the risk of cardiovascular mortality associated with aTRH in the US population is unknown. We aimed to assess the risk of cardiovascular disease (CVD) mortality associated with aTRH in the US population.
\end{abstract}

Methods: We analyzed data from 6357 adult hypertensive participants of the National Health and Nutrition Examination Survey (1988-1994 and 1999-2010) linked to the National Death Index. Based on presence of uncontrolled hypertension [blood pressure (BP) $\geq 140 / 90 \mathrm{mmHg}$ ] and the number of antihypertensives prescribed, we classified participants into the following groups: non-aTRH (BP < 140/90 $\mathrm{mmHg}$ and $\leq 3$ antihypertensives); controlled aTRH (BP $<140 / 90 \mathrm{mmHg}$ and $\geq 4$ antihypertensives); and uncontrolled aTRH (BP $\geq 140 / 90 \mathrm{mmHg}$ and $\geq 3$ antihypertensives).

Results: Of the 6357 participants, 1522 had aTRH, representing a US prevalence of 7.6 million. Of the participants with aTRH, 432 had controlled aTRH and 1090 had uncontrolled aTRH. During follow-up (median 6 years), there were 550 CVD deaths. The cumulative incidence of CVD mortality was significantly higher in the aTRH group compared with non-aTRH group (log-rank $p<0.001$ ). In fully adjusted models, aTRH was associated with a $47 \%$ higher risk of CVD mortality compared with the non-aTRH group [1.47 (1.1-1.96)]. Similar increase in risk of CVD mortality was noted across aTRH subgroups compared with the non-aTRH group: controlled aTRH [1.66 (1.03-2.68)] and uncontrolled aTRH [1.43 (1.05-1.94)]. Among non-aTRH subgroups, those on 3 antihypertensive medications had a 35\% increased risk of CVD mortality than those on $<3$ medications [1.35 (0.98-1.86)].

Conclusions: aTRH is a common condition, affecting approximately 7.6 million Americans. Regardless of BP control, people with aTRH remain at a higher risk of cardiovascular outcomes. The risk of cardiovascular disease mortality remains high among those with controlled BP on 3 medications (non-aTRH) or $\geq 4$ medications (controlled aTRH), groups not generally considered at high risk. Future risk reduction interventions should consider focusing on these high-risk groups.

Keywords: Hypertension, Anti-hypertensives, Cardiovascular mortality, All-cause mortality

\footnotetext{
* Correspondence: kkaczma4@jhmi.edu

${ }^{1}$ Department of Medicine, Johns Hopkins University School of Medicine, 600

N. Wolfe St, Baltimore, MD 21287, USA

Full list of author information is available at the end of the article
} 


\section{Key points}

Question: What is the risk of cardiovascular disease (CVD) mortality associated with apparent treatment resistant hypertension (aTRH) in the US population?

Findings: In this retrospective case-control study that included 6357 adults, aTRH was associated with a 47\% higher risk of cardiovascular mortality compared with the non-aTRH group. aTRH has a US prevalence of 7.6 million people.

Meaning: Regardless of BP control, people with aTRH remain at a higher risk of cardiovascular outcomes; future risk reduction interventions should consider focusing on these high-risk groups.

\section{Background}

Hypertension affects $29 \%$ of the US adult population, corresponding to approximately 90 million Americans [1]. Hypertension is a risk factor for a number of adverse cardiovascular outcomes, including stroke, coronary heart disease, and heart failure [2]. Treatment of hypertension prevents and reduces cardiovascular morbidity, notably a $40 \%$ reduction in risk of stroke and $15 \%$ reduction in risk of myocardial infarction [3, 4]. Recent data suggest that hypertension awareness has improved with a reduction in the proportion of untreated hypertensive patients; however, the percentage of patients on multiple antihypertensive medications has progressively risen [5].

Apparent treatment-resistant hypertension (aTRH) is defined as hypertension that remains uncontrolled $(\geq 140 / \geq 90 \mathrm{mmHg}$ ) despite treatment with $\geq 3$ antihypertensive medications or hypertension that is controlled $(<140 / 90 \mathrm{mmHg})$ on $\geq 4$ antihypertensives [6]. In comparison with non-aTRH hypertensive patients, patients with aTRH, regardless of blood pressure (BP) control, have a higher risk of coronary artery disease, cardiovascular disease, heart failure, end-stage renal disease (ESRD), and all-cause mortality [7-10]. With the rise in prevalence of hypertensive patients with aTRH from $5.5 \%$ in 1988 to $11.8 \%$ in 2008 , aTRH has become a common condition within the US population [5]. However, the risk of cardiovascular mortality associated with aTRH in the US population is unknown.

The goal of our study was to assess the risk of cardiovascular mortality amongst individuals with aTRH in the US using data from the National Health and Nutrition Examination Survey. Furthermore, we sought to compare the risk of cardiovascular mortality amongst aTRH subgroups, stratified by BP control.

\section{Methods}

\section{Study sample}

The NHANES are cross-sectional, multistage, stratified, clustered probability sample of the noninstitutionalized US civilian population conducted by the National Center for Health Statistics (NCHS), a branch of the Center for Disease Control and Prevention. NHANES III was conducted from 1988 to 1994 and the continuous NHANES were conducted from 1999 to 2010 with data released in 2 year cycles. We excluded participants that were $<18$ years of age, had no recorded BP measurements, no follow-up, no antihypertensive use, and no known diagnosis of hypertension (Fig. 1). We further excluded persons with inadequately treated uncontrolled hypertension defined as $\mathrm{BP} \geq 140 / 90 \mathrm{mmHg}$ and use of $<3$ antihypertensive medications because these patients are not considered to have aTRH based on current definitions of treatment resistant hypertension, but we cannot exclude the presence of aTRH given inadequate antihypertensive treatment. After these exclusions, 6357 participants were included in the final study population. The protocols for conduct of NHANES were approved by the NCHS institutional review board and informed consent was obtained from all participants. The Johns Hopkins Medical Institutions Institutional Review Board reviewed and approved the study.

\section{BP, Antihypertensives and definition of treatment resistant hypertension}

$\mathrm{BP}$ was measured after the participant had been resting in a seated position for $5 \mathrm{~min}$ with feet on the floor. Three consecutive BP readings were obtained and reported as the mean of all systolic readings over the mean of all diastolic readings [11]. Antihypertensive medication use was self-reported, and antihypertensive medications were classified as diuretics, angiotensin converting enzyme inhibitors, angiotensin receptor blockers, beta blockers, calcium channel blockers, and others according to classification codes provided with the NHANES data. Medication dose data was not available. We defined uncontrolled hypertension as systolic BP $\geq 140 \mathrm{mmHg}$ and/or diastolic BP $\geq 90 \mathrm{mmHg}$. Using BP control and number of antihypertensive medications, we classified participants into the following groups:

a) Non-aTRH: Hypertensive participants with controlled hypertension (BP $<140 /<90 \mathrm{mmHg}$ ) treated with $\leq 3$ medications. We further subdivided this group into those on 3 antihypertensives and those on $<3$ antihypertensives.

b) Controlled aTRH: Hypertensive participants with controlled hypertension (BP $<140 /<90 \mathrm{mmHg}$ ) treated with $\geq 4$ antihypertensives.

c) Uncontrolled aTRH: Hypertensive participants with uncontrolled hypertension (BP $\geq 140 / 90 \mathrm{mmHg}$ ) treated with $\geq 3$ antihypertensives.

\section{Measurements - other variables}

Standardized questionnaires were administered at home, and physical examination and laboratory test specimen 


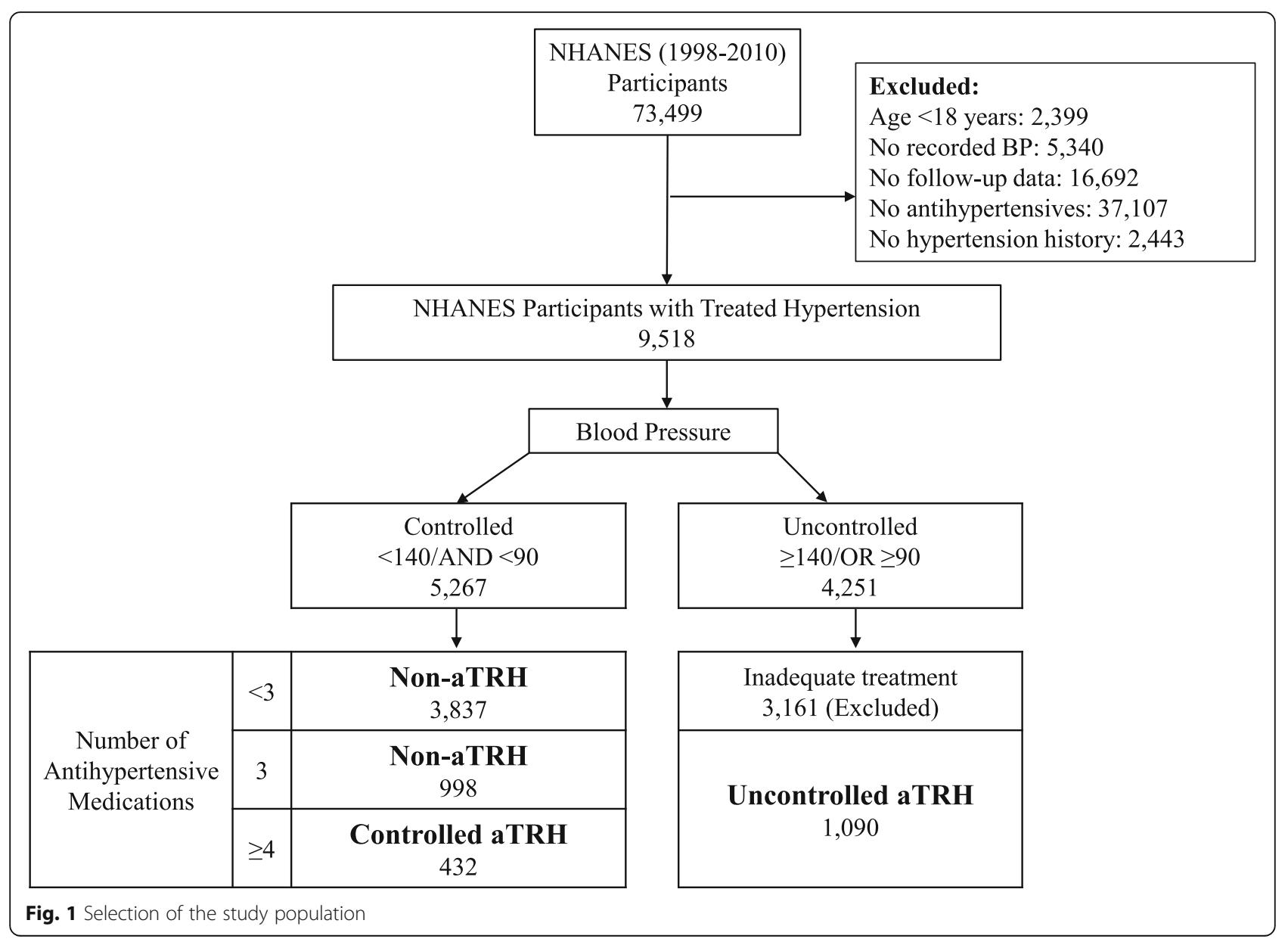

collection were performed at the mobile examination center. Self-reported race/ethnicity was categorized as non-Hispanic White, non-Hispanic Black, MexicanAmerican or other. Smoking was defined as either ever smoked or never having smoked. Participants were considered to have diabetes mellitus if they reported being told by a doctor that they had diabetes at a time other than pregnancy or if they were taking insulin or oral hypoglycemic agents. Cardiovascular disease was considered to be present at baseline if the participant reported being informed by a doctor of prior heart attack, congestive heart failure or stroke. Body mass index was calculated by dividing weight in $\mathrm{kg}$ by height in $\mathrm{m}^{2}$ and modeled as a linear spline with knots at $18 \mathrm{~kg} / \mathrm{m}^{2}$ and $25 \mathrm{~kg} / \mathrm{m}^{2}$. Serum creatinine was measured using a kinetic rate Jaffe method and used to calculate estimated glomerular filtration rate (eGFR) [12], and modeled as a linear spline with knots at 60 and $90 \mathrm{ml} / \mathrm{min} / 1.73 \mathrm{~m}^{2}$. Total cholesterol levels included both fasting and non-fasting samples and were modeled as a continuous variable. C-reactive protein was measured by latex-enhanced nephelometry (Dade Behring). Urinary albumin was measured by solid-phase fluorescence immunoassay, and urinary creatinine was measured by the modified kinetic method of Jaffe using a Beckman Coulter Synchron AS/ Astra Analyzer (Beckman Coulter, Inc., Fullerton, California). C-reactive protein and urinary albumin-tocreatinine ratio were modeled as continuous variables after natural log transformation.

\section{Causes of death}

Causes of death were obtained using the NHANES Public-use Linked Mortality Files [11]. This file contains mortality follow-up data on NHANES participants obtained via National Death Index (NDI) linkage through December 31, 2011. The underlying cause of death was coded using the UCOD_LEADING variable and was classified as either all-cause death or death due to cardiovascular disease (UCOD_LEADING values 001 or 005) [11].

\section{Statistical analysis}

All analyses were performed incorporating sampling that account for the complex survey design of NHANES, allowing generation of nationally representative estimates for the US population. Population estimates were 
standardized to the 2017 U.S. population. Baseline characteristics were compared among those with controlled hypertension versus aTRH and among subgroups of aTRH. Survival analysis techniques were used to analyze the risk of mortality associated with aTRH. Individuals who were alive on December 31, 2011 were censored in the analyses. Kaplan-Meier survival plots were used to assess the differences in survival among those with controlled hypertension and aTRH categories. Poisson regression was used to calculate mortality rates. Modified Cox proportional hazards regression was used to model the risk of death with non-aTRH participants as the reference group. Hazard ratios (HR) were calculated for unadjusted models (Model 1) and after sequentially adjusting for age, race/ethnicity, sex (Model 2), followed by further adjustment for diabetes and cardiovascular disease, smoking status, body mass index, serum total cholesterol, and C-reactive protein (CRP) (Model 3). The fully adjusted model (Model 4) further adjusted for estimate glomerular filtration rate (eGFR) and albuminto-creatinine ratio (ACR). Pre-specified subgroup analyses included categories of age, sex, race/ethnicity, smoking status, diabetes, cardiovascular disease, diuretic use, eGFR, CRP and ACR. All analyses were performed using survey (svy) commands in Stata 13.1 (Stata Corp, www.stata.com). A $p$-value of $<0.05$ was considered statistically significant.

\section{Results}

\section{Baseline participant characteristics}

Figure 1 describes the selection of the final study population. Of the 6357 NHANES participants included in this analysis, 1522 had aTRH. Within the US population, this corresponds to a prevalence of 7.6 million adults with aTRH, of which 2.27 million have controlled aTRH, and 5.33 million have uncontrolled aTRH (Table 1). The prevalence of hypertensive US adults without aTRH is 29.04 million, of which 23.49 million have controlled BP on $<3$ medications, and 5.55 million have controlled BP on 3 medications. Note that these prevalence estimates exclude people with inadequately treated (uncontrolled on $<3$ medications) or untreated hypertension. The baseline characteristics of the hypertensive NHANES participants with and without aTRH are described in Table 1. Compared to the participants without aTRH, those with aTRH were older (66 years versus 59 years) and more likely to be African-American, have a history of diabetes and prior cardiovascular disease, and have a lower eGFR. Among aTRH versus non-aTRH participants, $86.8 \%$ vs. $46.3 \%$ were on a diuretic, $83.2 \%$ vs. $63.4 \%$ were on an ACE-I or ARB, $56.6 \%$ vs. $24.5 \%$ were on a calcium channel blocker, and $66.6 \%$ vs. $34.6 \%$ were on a beta blocker.
Baseline characteristics were similar between aTRH subgroups. Notably, the controlled aTRH subgroup had a greater percentage of participants with prior CVD (48.5\% vs. $36.4 \%$ ), defined as prior heart attack, congestive heart failure, or stroke, compared to the uncontrolled aTRH subgroup. Compared to non-aTRH patients on $<3$ medications, patients on 3 medications were more likely to have diabetes $(32.4 \%$ vs. $22.7 \%)$, prior CVD (31.4\% vs. $15.9 \%)$, and a prescription for a diuretic ( $83.5 \%$ vs. $37.5 \%)$.

\section{aTRH and risk of cardiovascular mortality}

There were 541 deaths due to cardiovascular disease during follow-up (median 6 years). The crude cardiovascular mortality rate (Table 2) was over 2-fold higher in the aTRH group (19.4 per 1000 person-years) compared with the non-aTRH group (7.4 deaths per 1000 person-years). Within the aTRH subgroups, the crude cardiovascular mortality was similar between the controlled aTRH subgroup (19.1 per 1000 person-years) and uncontrolled aTRH subgroup (19.5 per 1000 person-years). Within the non-aTRH subgroups, the crude cardiovascular mortality was 2 -fold greater in the participants on 3 medications (13 per 1000 person-years) in comparison to those on $<3$ medications (6.3 per 1000 person-years).

The cumulative cardiovascular mortality in the nonaTRH and aTRH groups is displayed in Fig. 2. Those with aTRH, both controlled and uncontrolled, had a significantly higher cardiovascular mortality than those in the non-aTRH groups (log-rank $p<0.001)$.

The unadjusted and adjusted models for risk of cardiovascular mortality are presented in Table 2. In Model 3, adjusting for age, race/ethnicity, sex, body mass index, history of diabetes and cardiovascular disease, smoking status, and serum total cholesterol and CRP, people with aTRH had a 73\% higher risk of cardiovascular mortality compared with the non-aTRH group [1.73 (1.34-2.23)]. After further adjustment (Model 4), accounting for eGFR and ACR (Table 2), people with aTRH had a $47 \%$ higher risk of cardiovascular mortality compared with the non-aTRH group [1.47 (1.1-1.96)]. Similarly, aTRH subgroups had a higher risk of cardiovascular mortality in comparison to the non-aTRH group: controlled aTRH [1.66 (1.03-2.68)] and uncontrolled aTRH [1.43 (1.051.94)]. Among non-aTRH subjects, those on 3 antihypertensives had a trend toward greater risk of cardiovascular mortality than those on $<3$ antihypertensives [1.35 (0.981.86)] (Table 3).

\section{All-cause mortality}

In Model 3, people with aTRH had a 33\% increased risk of all-cause mortality compared with the non-aTRH group [1.33 (1.13-1.57)]. In the fully adjusted model (Model 4), there was no significant increased risk of all-cause mortality of the aTRH group in comparison to 


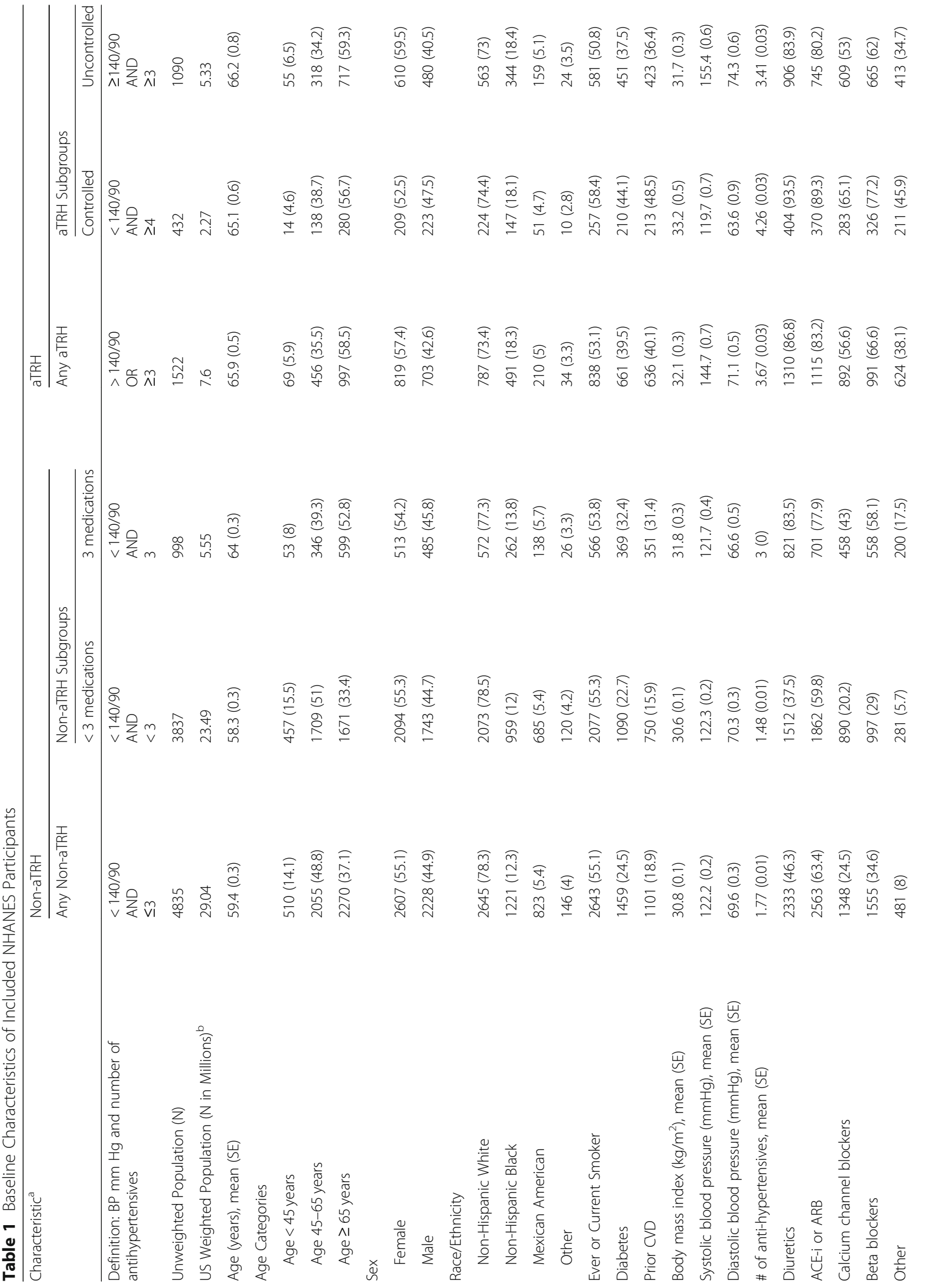




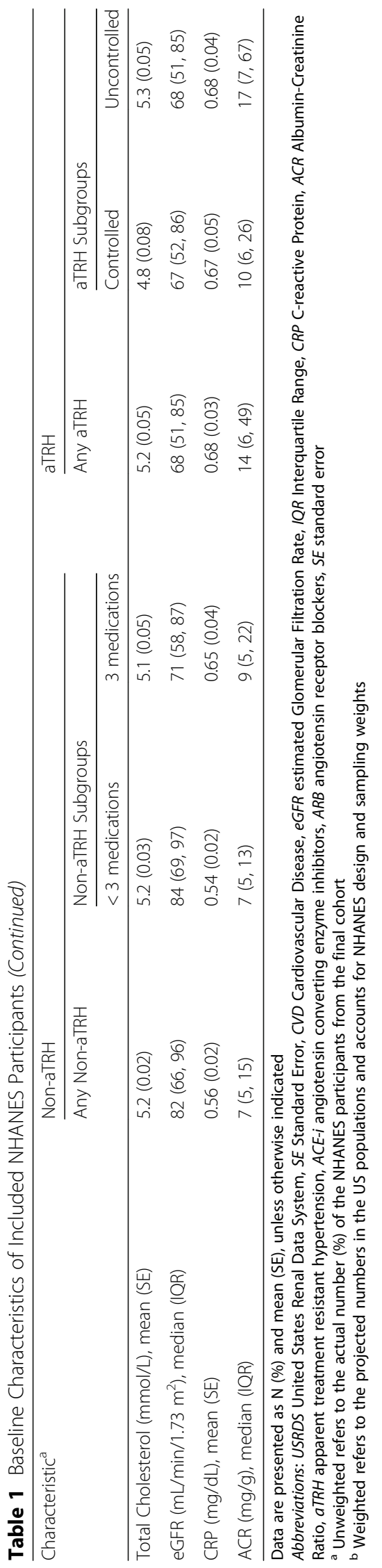




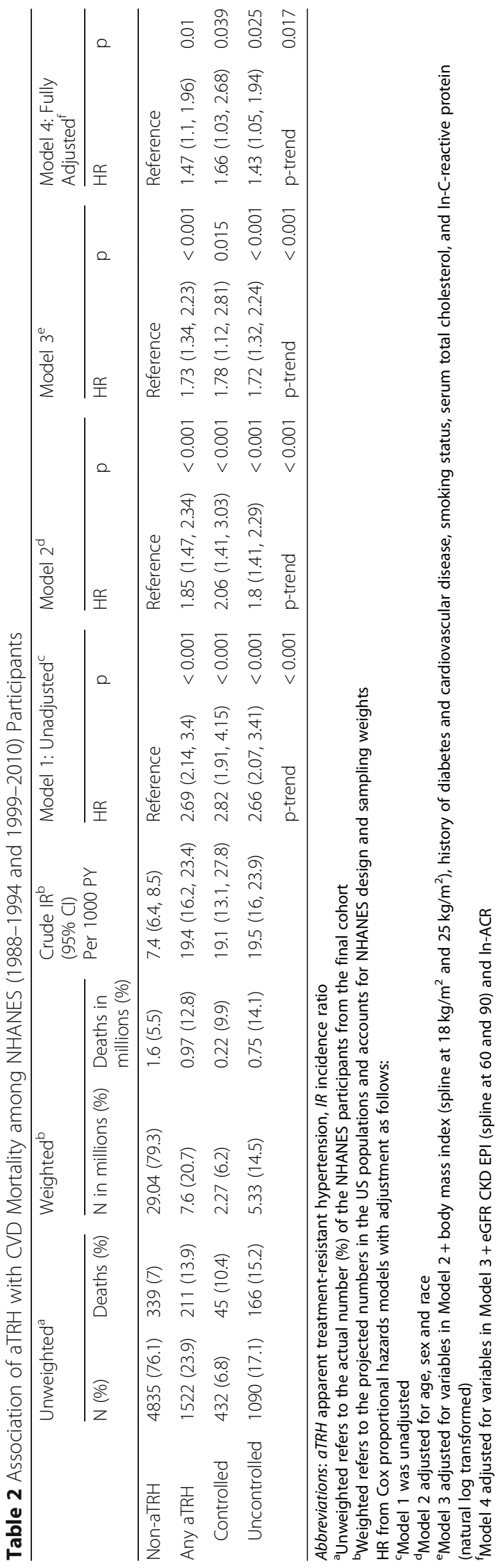




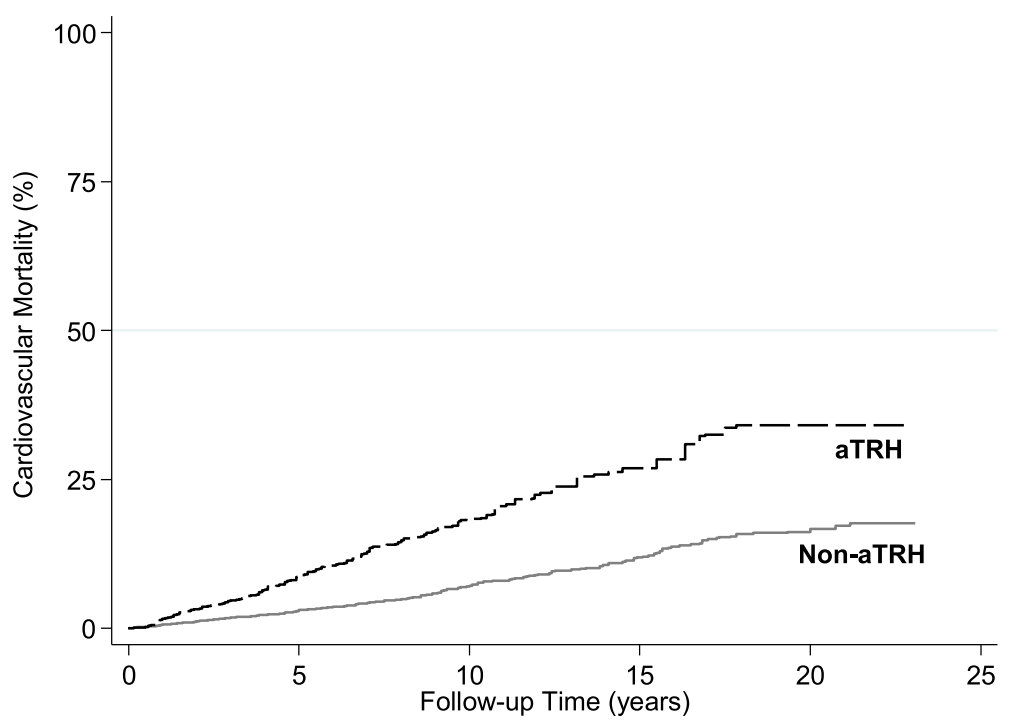

Fig. 2 Cumulative Incidence of Cardiovascular Disease Mortality among NHANES participants with aTRH and controlled hypertension

the non-aTRH group $[1.15 \quad(0.97-1.36)]$ (Additional file 1: Table S1).

\section{Subgroup analyses}

Pre-specified subgroup analyses are presented in Table 4. Due to multiple comparisons, a p-interaction of $<0.005$ $(p=0.05 / 10)$ is suggested as a significant value. Using this threshold, there were no significant differences within subgroups.

\section{Discussion}

In this U.S. nationally-representative study, we found that 7.6 million US adults with diagnosed and treated hypertension had aTRH, which was associated with $47 \%$ higher risk of cardiovascular mortality compared to people without aTRH. Those with controlled aTRH and uncontrolled aTRH had 66 and 43\% higher risk of cardiovascular death, compared with the non-aTRH group. Among participants without aTRH, those on 3 antihypertensive medications had a trend toward higher risk of cardiovascular mortality than those on $<3$ antihypertensive medications. Our findings suggest that patients with aTRH and non-aTRH requiring 3 medications for BP control represent high cardiovascular disease risk groups and should be considered as targets for risk reduction interventions.

Hypertension increases the risk of coronary artery disease, myocardial infarction, stroke, accelerated atherosclerosis, congestive heart failure, and ESRD [13]. While microvascular changes play a role in the pathogenesis of hypertension, uncontrolled hypertension is known to cause further microvascular structural and functional alterations, notably smooth muscle cell hypertrophy and collagen deposition in resistance arterioles [14].
Hypertension is also associated with conditions, such as obesity and diabetes mellitus, that further alter and damage microvasculature in presence of an inflammatory milieu [15], resulting in impaired endothelial insulin signaling and insulin-stimulated nitric oxide synthesis [16]. Similarly, the aTRH participants in our study were more likely to have diabetes $(39.5 \%$ vs. $24.5 \%)$ and CVD $(40.1 \%$ vs. $18.9 \%)$ than the non-aTRH participants. These comorbidities may have influenced the hypertensive disease burden and blood pressure control in aTRH participants. It is important to consider the differences in the baseline characteristics between the aTRH and non-aTRH groups when interpreting our results. However, the baseline characteristics seen in our study are representative of that of the US population, thus the findings of our study are applicable to general practice.

Over the past three decades, significant efforts have been focused on improving awareness, treatment, and control of hypertension. According to Egan et al., the percentage of hypertensive patients with uncontrolled hypertension decreased from $73.2 \%$ in 1988 to $52.5 \%$ in 2008 [5]. However, with the reduction in uncontrolled hypertension and increased awareness of treatment, there has been an increase in the prevalence of aTRH. Between 1988 and 2008, the percentage of hypertensive patients on 1 or 2 medications remained relatively constant, while the percentage of patients on $\geq 3$ medications increased progressively [5]. It is generally assumed that once the BP is at goal, regardless of the number of medications being used, the risk of cardiovascular disease is mitigated. However, our study demonstrates that despite adequate blood pressure control, people with aTRH are at an increased risk of cardiovascular mortality. 


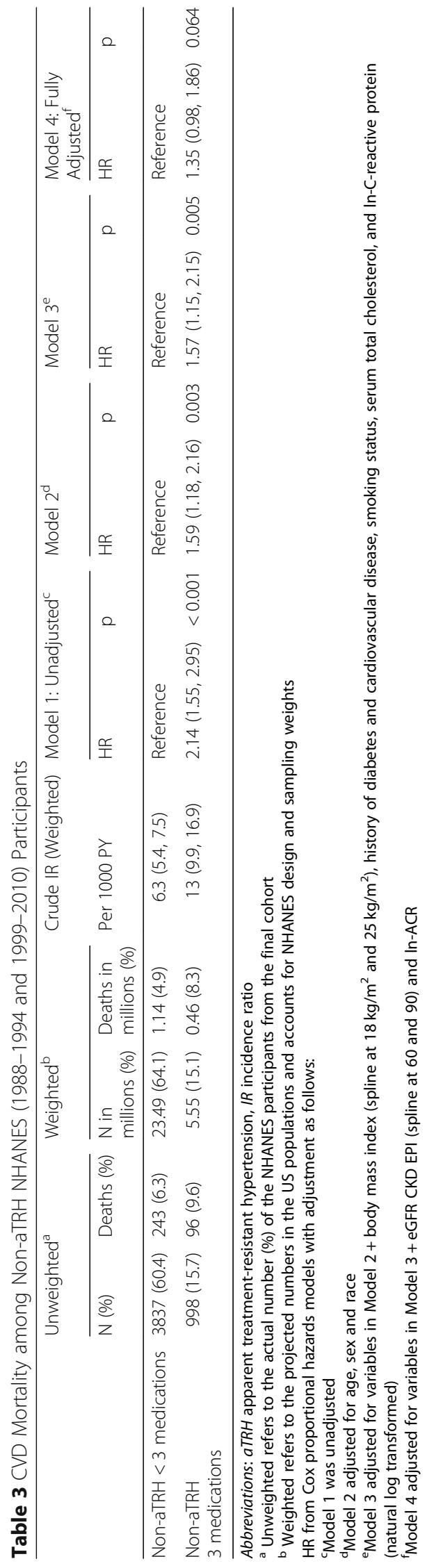


Table 4 Subgroup Analysis for Cardiovascular Mortality Associated with aTRH

\begin{tabular}{|c|c|c|c|c|}
\hline & N & Deaths & Any aTRH vs. Controlled & $\mathrm{p}$-interaction \\
\hline \multicolumn{5}{|l|}{ Age (years) } \\
\hline$<45$ years & 579 & 15 & NA & \multirow[t]{3}{*}{0.211} \\
\hline $45-65$ years & 2511 & 117 & $2.13(1.18,3.88)$ & \\
\hline$\geq 65$ years & 3267 & 418 & $1.23(0.91,1.66)$ & \\
\hline \multicolumn{5}{|l|}{ Sex } \\
\hline Female & 3426 & 269 & $1.87(1.26,2.79)$ & \multirow[t]{2}{*}{0.033} \\
\hline Male & 2931 & 281 & $1.13(0.76,1.69)$ & \\
\hline \multicolumn{5}{|l|}{ Race/Ethnicity } \\
\hline Non-Hispanic White & 3432 & 343 & $1.56(1.13,2.15)$ & \multirow{4}{*}{0.583} \\
\hline Non-Hispanic Black & 1712 & 143 & $1.17(0.73,1.88)$ & \\
\hline Mexican American & 1033 & 58 & $1.15(0.38,3.43)$ & \\
\hline Other & 180 & 6 & NA & \\
\hline Nonsmoker & 2869 & 218 & $2.16(1.34,3.47)$ & \multirow[t]{2}{*}{0.075} \\
\hline Smoker & 3481 & 332 & $1.19(0.85,1.65)$ & \\
\hline No Diabetes & 4237 & 349 & $1.48(1.05,2.07)$ & \multirow[t]{2}{*}{0.746} \\
\hline Diabetes & 2120 & 201 & $1.46(0.94,2.28)$ & \\
\hline No CVD & 4558 & 261 & $1.68(1.1,2.56)$ & \multirow[t]{2}{*}{0.03} \\
\hline CVD & 1737 & 282 & $1.35(1,1.81)$ & \\
\hline No Diuretic & 2714 & 161 & $1.223(0.62,2.4)$ & \multirow[t]{2}{*}{0.818} \\
\hline Diuretic & 3643 & 389 & $1.48(1.04,2.11)$ & \\
\hline No ACE-I or ARB & 1705 & 101 & $1.35(0.69,2.62)$ & \multirow[t]{2}{*}{0.562} \\
\hline ACE-I or ARB & 3678 & 260 & $1.7(1.18,2.44)$ & \\
\hline No $C C B^{b}$ & 4117 & 303 & $1.54(0.98,2.43)$ & \multirow[t]{2}{*}{0.549} \\
\hline$C C B^{b}$ & 2240 & 247 & $1.35(0.93,1.98)$ & \\
\hline No Betablocker & 3811 & 309 & $1.51(1.03,2.22)$ & \multirow[t]{2}{*}{0.411} \\
\hline Betablocker & 2546 & 241 & $1.4(0.91,2.16)$ & \\
\hline No Antihtn_other ${ }^{c}$ & 5252 & 411 & $1.41(0.98,2.01)$ & \multirow[t]{2}{*}{0.649} \\
\hline Antihtn_other ${ }^{c}$ & 1105 & 139 & $1.55(0.89,2.69)$ & \\
\hline \multicolumn{5}{|l|}{ eGFR CKDEPI Categories ${ }^{a}$} \\
\hline$\geq 120$ & 135 & 5 & NA & \multirow[t]{6}{*}{0.521} \\
\hline $90-119$ & 1617 & 47 & $1.6(0.57,4.47)$ & \\
\hline $60-89$ & 2677 & 215 & $1.34(0.88,2.04)$ & \\
\hline $45-59$ & 929 & 129 & $1.94(1.17,3.2)$ & \\
\hline $30-44$ & 417 & 76 & $1.08(0.47,2.47)$ & \\
\hline$<30$ & 187 & 38 & $2.33(0.32,16.86)$ & \\
\hline \multicolumn{5}{|l|}{$\mathrm{CRP}(\mathrm{mg} / \mathrm{dL})$} \\
\hline$<0.22$ & 2546 & 220 & $1.18(0.76,1.84)$ & \multirow[t]{3}{*}{0.582} \\
\hline $0.22-0.99$ & 2487 & 187 & $1.74(1.16,2.6)$ & \\
\hline$\geq 1.00$ & 981 & 109 & $2.17(0.99,4.77)$ & \\
\hline \multicolumn{5}{|l|}{$A C R$} \\
\hline$<30$ & 4807 & 310 & $1.79(1.27,2.52)$ & \multirow[t]{3}{*}{0.063} \\
\hline $30-300$ & 1061 & 142 & $1.31(0.83,2.07)$ & \\
\hline$\geq 300$ & 292 & 57 & $0.93(0.43,2)$ & \\
\hline
\end{tabular}

Abbreviations: aTRH apparent treatment-resistant hypertension, CVD Cardiovascular Disease, eGFR CKD-EPI estimated Glomerular Filtration Rate using Chronic Kidney Disease Epidemiology Collaboration, CRP C-reactive Protein, ACR Albumin-Creatinine Ratio

a measured in $\mathrm{mL} / \mathrm{min} / 1.73 \mathrm{~m}^{2}$

${ }^{b}$ Calcium channel blocker

c Other anti-hypertensive not included in the preceding anti-hypertensive classes 
In prior studies, aTRH was found to be associated with higher risk of ESRD, ischemic cardiac events, congestive heart failure, and stroke in comparison to patients with non-resistant hypertension [7, 17]. Importantly, cardiovascular disease risk remains elevated amongst patients with aTRH, regardless of BP control [7, 17]. In a study by Muntner et al., 14,684 ALLHAT trial participants were categorized as having aTRH or non-resistant hypertension. There was a higher risk of coronary heart disease, stroke, all-cause mortality, heart failure, and ESRD in those with aTRH [7]. Similarly, in a study by Irvin et al., a higher risk of coronary heart disease, stroke, and all-cause mortality was noted among those with aTRH [18]. Our study similarly demonstrates increased risk of CVD mortality amongst people with aTRH, therefore extending the findings from prior studies with data generalizable to the US population. However, unlike prior studies $[8-10,18]$, our study did not show a significantly increased risk of all-cause mortality in the aTRH group in comparison to the non-aTRH group. This suggests that individuals with aTRH are at greater risk of cardiovascular-related mortality but overall have similar risk of all-cause mortality.

Although the pathophysiology of aTRH is not well-understood, it is known that patients with aTRH have higher levels of brain-type and natriuretic peptides as well as aldosterone [19]. Additional contributing factors to aTRH may include non-adherence with lifestyle changes, including diet and exercise, and inadequate pharmacologic therapy [20]. Pharmacologic management of apparent treatment-resistant hypertension should include therapeutic optimization of antihypertensive dosages, systematic measurement of BP response to treatment, and assistance in overcoming barriers to adherence. Our study shows that even with medication prescription and BP control, aTRH patients remain at a higher risk of cardiovascular outcomes, suggesting a need for further risk mitigation strategies. The ongoing TRIUMPH trial (Clinicaltrials.gov no. NCT02342808) is evaluating the efficacy of lifestyle intervention using sodium restriction, DASH diet, exercise, weight management, and patient instruction and education in the treatment of aTRH [21]. Experimental treatments include renal denervation and electrical carotid baroreceptor stimulation using implantable devices; however, further studies are necessary for determination of efficacy and safety for use in treatment of aTRH $[22,23]$.

The observed associations between aTRH and cardiovascular mortality in our study provides some interesting insights (Table 2). In the unadjusted model (Model 1), aTRH was associated with a 2.5 -fold higher risk of cardiovascular mortality, compared to the non-aTRH group. Adjusting for age, sex, and race (Model 2) reduces the magnitude of this association, reflecting the confounding effects of these variables. The association is further attenuated by adjusting for additional confounders in Model 3, in particular baseline diabetes and cardiovascular disease which had higher prevalence in the aTRH group as compared to the non-aTRH group. In Model 4, adjusting for kidney function (estimated glomerular filtration rate and albuminuria), further attenuates the association. It is likely that some of these adjustment factors, such as baseline cardiovascular disease and kidney function mediate the observed association between aTRH and outcomes. The higher systolic BP in the aTRH group (144 $\mathrm{mmHg}$ ) as compared to the non-aTRH group $(122 \mathrm{mmHg})$ could be contributing to higher prevalence of cardiovascular and kidney disease noted at the baseline in the aTRH group (Table 1). At baseline $40.1 \%$ of those in the aTRH group had cardiovascular disease, compared to $18.9 \%$ in the non-aTRH group. Similarly, the aTRH group had lower estimated glomerular filtration rate $\left(68 \mathrm{ml} / \mathrm{min} \mid 1.73 \mathrm{~m}^{2}\right)$ as compared to the non-aTRH group $\left(82 \mathrm{ml} / \mathrm{min} \mid 1.73 \mathrm{~m}^{2}\right)$. Clinical implication of these findings is that in patients with aTRH (whether controlled or uncontrolled) careful attention must be paid to reducing cardiovascular risk and preserving kidney function. From a population health perspective, aTRH identifies a patient population at 2.5 -fold higher risk of cardiovascular mortality. This high-risk population can be easily identified using electronic health records, and population health management strategies could target this population for focused interventions such as lifestyle modification, and use of medications with cardioprotective and renoprotective effects.

Several limitations of our study deserve mention. First, we only had participant reported prescriptions and did not have information on prescription adherence which limits our ability to differentiate between true treatment-resistant hypertension and uncontrolled hypertension as the result of medication noncompliance [24]. Second, medications are assessed at a single timepoint due to the cross-sectional nature of NHANES. A time-updated analysis could account for changing patterns of comorbidities, medications, and BP over time and may find different associations. Third, we did not have information on medication doses. Physician prescription patterns, such as the use of low dose combination antihypertensive medications, may incorrectly assign participants to the aTRH category and bias the observed associations. Fourth, we only assessed cardiovascular mortality and did not have information on cardiovascular events. These limitations of our study are balanced by its strengths including its large sample size representative of the U.S. population, inclusion of racial/ ethnic minorities, broad age range, rigorous data collection and extensive information on covariates, large 
number of events, and near-complete mortality follow-up using the NDI. The results of our study are generalizable to non-institutionalized U.S. adults.

\section{Conclusions}

In conclusion, in the US population, aTRH is common with 7.6 million affected adults and is associated with higher risk of cardiovascular disease mortality. In particular, the risk of cardiovascular disease mortality remains high among those with controlled BP on 3 medications (non-aTRH) or $\geq 4$ medications (controlled aTRH), groups not generally considered at high risk. Future risk reduction interventions should consider focusing on these high-risk groups.

\section{Additional file}

Additional file 1: Table S1. Cumulative Incidence of All-Cause Mortality among NHANES participants with aTRH and controlled hypertension. (DOCX $16 \mathrm{~kb}$ )

\section{Acknowledgements}

Not applicable.

\section{Funding}

Dr. Shafi was supported by R03-DK-104012 and R01-HL-132372-01.

\section{Availability of data and materials}

Not applicable.

\section{Authors' contributions}

KRK and TS developed the study design. JC and YS performed the statistical analyses. All authors assisted with interpretation of data. KRK, TS, and SS drafted the manuscript, and all authors reviewed and approved the manuscript.

\section{Ethics approval and consent to participate}

The protocols for conduct of NHANES were approved by the NCHS institutional review board and written informed consent was obtained from all participants. The Johns Hopkins Medical Institutions Institutional Review Board reviewed and approved the study.

\section{Consent for publication}

Not applicable.

\section{Competing interests}

Tariq Shafi is an Editorial Board Member of BMC Nephrology.

\section{Publisher's Note}

Springer Nature remains neutral with regard to jurisdictional claims in published maps and institutional affiliations.

\section{Author details}

'Department of Medicine, Johns Hopkins University School of Medicine, 600 N. Wolfe St, Baltimore, MD 21287, USA. Welch Center for Prevention, Epidemiology and Clinical Research, Johns Hopkins Medical Institutions, Baltimore, MD, USA. ${ }^{3}$ Department of Biostatistics, Johns Hopkins Bloomberg School of Public Health, Baltimore, MD, USA. ${ }^{4}$ Department of Medicine, University of Mississippi Medical Center, Jackson, MS, USA.
Received: 23 October 2017 Accepted: 29 March 2019

Published online: 25 April 2019

\section{References}

1. Yoon SS, Carroll MD, Fryar CD. Hypertension prevalence and control among adults: United states, 2011-2014. NCHS data brief; 2015. p. 1-8.

2. He J, Whelton PK. Elevated systolic blood pressure and risk of cardiovascular and renal disease: overview of evidence from observational epidemiologic studies and randomized controlled trials. Am Heart J. 1999:138:211-9.

3. Whitworth JA. 2003 world health organization (who)/international society of hypertension (ish) statement on management of hypertension. J Hypertens. 2003;21:1983-92.

4. Collins R, Peto R, MacMahon S, Hebert P, Fiebach NH, Eberlein KA, Godwin J, Qizilbash N, Taylor JO, Hennekens CH. Blood pressure, stroke, and coronary heart disease. Part 2, short-term reductions in blood pressure: overview of randomised drug trials in their epidemiological context. Lancet. 1990;335:827-38.

5. Egan BM, Zhao Y, Axon RN, Brzezinski WA, Ferdinand KC. Uncontrolled and apparent treatment resistant hypertension in the United States, 1988 to 2008. Circulation. 2011:124:1046-58.

6. Calhoun DA, Jones D, Textor S, Goff DC, Murphy TP, Toto RD, White A, Cushman WC, White W, Sica D, Ferdinand K, Giles TD, Falkner B, Carey RM. Resistant hypertension: diagnosis, evaluation, and treatment: a scientific statement from the american heart association professional education committee of the council for high blood pressure research. Circulation. 2008:117:e510-26.

7. Muntner P, Davis BR, Cushman WC, Bangalore S, Calhoun DA, Pressel SL, Black HR, Kostis JB, Probstfield JL, Whelton PK, Rahman M. Treatment-resistant hypertension and the incidence of cardiovascular disease and end-stage renal disease: results from the antihypertensive and lipid-lowering treatment to prevent heart attack trial (allhat). Hypertension. 2014;64:1012-21.

8. Smith SM, et al. Mortality risk associated with resistant hypertension among women: analysis from three prospective cohorts encompassing the Spectrum of Women's heart disease. J Women's Health (2002). 2016;25(10): 996-1003.

9. Smith SM, et al. Cardiovascular and mortality risk of apparent resistant hypertension in women with suspected myocardial ischemia: a report from the NHLBI-sponsored WISE study. J Am Heart Assoc. 3,1 e000660. 28 Feb. 2014. https://doi.org/10.1161/JAHA.113.000660.

10. Bangalore $S$, et al. Prevalence, predictors, and outcomes in treatmentresistant hypertension in patients with coronary disease. Am J Med. 2014; 127:71-81.

11. Centers for Disease Control and Prevention (CDC). National Center for Health Statistics (NCHS). National Health and Nutrition Examination Survey Data. Hyattsville, MD: U.S. Department of Health and Human Services, Centers for Disease Control and Prevention, 2018. https://wwwn.cdc.gov/ nchs/nhanes/analyticguidelines.aspx.

12. Levey AS, Stevens LA, Schmid CH, Zhang YL, Castro AF, 3rd, Feldman H Kusek JW, Eggers P, Van Lente F, Greene T, Coresh J. A new equation to estimate glomerular filtration rate. Ann Intern Med 2009:150:604-612.

13. Lerman LO, Textor SC. Hypertension in 2015: resistant hypertension: impact and evolving treatment options. Nat Rev Nephrol. 2016;12:70-2.

14. Rizzoni D, Agabiti-Rosei E. Structural abnormalities of small resistance arteries in essential hypertension. Intern Emerg Med. 2012;7:205-12.

15. Scalia R. The microcirculation in adipose tissue inflammation. Rev Endocr Metab Disord. 2013;14:69-76

16. Yudkin JS, Eringa E, Stehouwer CD. "Vasocrine" signalling from perivascular fat: a mechanism linking insulin resistance to vascular disease. Lancet. 2005; 365:1817-20.

17. Sim JJ, Bhandari SK, Shi J, Reynolds K, Calhoun DA, Kalantar-Zadeh K, Jacobsen SJ. Comparative risk of renal, cardiovascular, and mortality outcomes in controlled, uncontrolled resistant, and nonresistant hypertension. Kidney Int. 2015;88:622-32.

18. Irvin MR, Booth JN 3rd, Shimbo D, Lackland DT, Oparil S, Howard G, Safford MM, Muntner P, Calhoun DA. Apparent treatment-resistant hypertension and risk for stroke, coronary heart disease, and all-cause mortality. J Am Soc Hypertens. 2014;8:405-13.

19. Gaddam KK, Nishizaka MK, Pratt-Ubunama MN, Pimenta E, Aban I, Oparil S, Calhoun DA. Characterization of resistant hypertension: association between resistant hypertension, aldosterone, and persistent intravascular volume expansion. Arch Intern Med. 2008;168:1159-64. 
20. Braam B, Taler SJ, Rahman M, Fillaus JA, Greco BA, Forman JP, Reisin E, Cohen DL, Saklayen MG, Hedayati SS. Recognition and management of resistant hypertension. Clin J Am Soc Nephrol. 2017;12(3):524-35.

21. Blumenthal JA, Sherwood A, Smith PJ, Mabe S, Watkins L, Lin PH, Craighead LW, Babyak M, Tyson C, Young K, Ashworth M, Kraus W, Liao L, Hinderliter A. Lifestyle modification for resistant hypertension: the triumph randomized clinical trial. Am Heart J. 2015;170:986-94 e985.

22. Bisognano JD, Bakris G, Nadim MK, Sanchez L, Kroon AA, Schafer J, de Leeuw PW, Sica DA. Baroreflex activation therapy lowers blood pressure in patients with resistant hypertension: Results from the double-blind, randomized, placebo-controlled rheos pivotal trial. J Am Coll Cardiol. 2011; 58:765-73.

23. Shafi T, Chacko M, Berger Z, Wilson LM, Gayleard J, Bass EB, Sozio SM. Ahrq technology assessments. In: Renal denervation in the medicare population. Rockville: Agency for Healthcare Research and Quality (US); 2016.

24. Beaussier H, Boutouyrie P, Bobrie G, Frank M, Laurent S, Coudore F, Azizi M. True antihypertensive efficacy of sequential nephron blockade in patients with resistant hypertension and confirmed medication adherence. J Hypertens. 2015;33:2526-33.

Ready to submit your research? Choose BMC and benefit from:

- fast, convenient online submission

- thorough peer review by experienced researchers in your field

- rapid publication on acceptance

- support for research data, including large and complex data types

- gold Open Access which fosters wider collaboration and increased citations

- maximum visibility for your research: over $100 \mathrm{M}$ website views per year

At $\mathrm{BMC}$, research is always in progress.

Learn more biomedcentral.com/submissions 